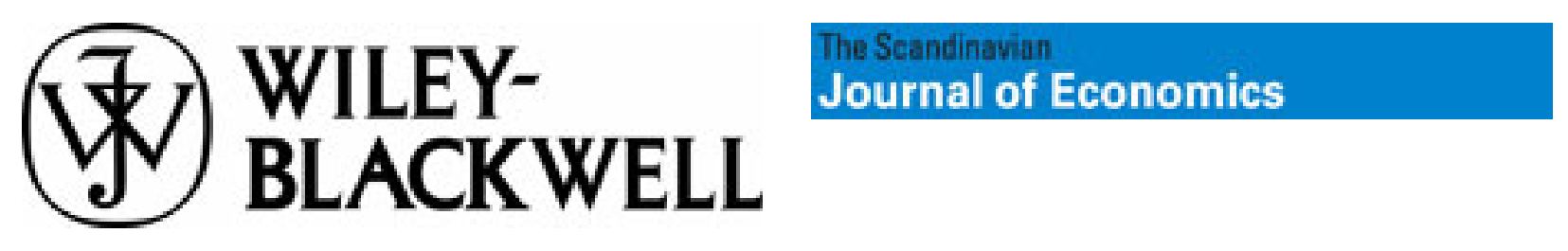

Exercises in Conjectural Equilibria

Author(s): Frank H. Hahn

Source: The Scandinavian Journal of Economics, Vol. 79, No. 2, Topics in Disequilibrium

Economics (1977), pp. 210-226

Published by: Blackwell Publishing on behalf of The Scandinavian Journal of Economics

Stable URL: http://www.jstor.org/stable/3439508

Accessed: 10/12/2010 20:31

Your use of the JSTOR archive indicates your acceptance of JSTOR's Terms and Conditions of Use, available at http://www.jstor.org/page/info/about/policies/terms.jsp. JSTOR's Terms and Conditions of Use provides, in part, that unless you have obtained prior permission, you may not download an entire issue of a journal or multiple copies of articles, and you may use content in the JSTOR archive only for your personal, non-commercial use.

Please contact the publisher regarding any further use of this work. Publisher contact information may be obtained at http://www.jstor.org/action/showPublisher?publisherCode=black.

Each copy of any part of a JSTOR transmission must contain the same copyright notice that appears on the screen or printed page of such transmission.

JSTOR is a not-for-profit service that helps scholars, researchers, and students discover, use, and build upon a wide range of content in a trusted digital archive. We use information technology and tools to increase productivity and facilitate new forms of scholarship. For more information about JSTOR, please contact support@ jstor.org.

Blackwell Publishing and The Scandinavian Journal of Economics are collaborating with JSTOR to digitize, preserve and extend access to The Scandinavian Journal of Economics. 


\title{
EXERCISES IN CONJECTURAL EQUILIBRIA
}

\author{
Frank H. Hahn \\ University of Cambridge, Cambridge, England
}

\begin{abstract}
In this paper one considers an economy in which individuals can transact at "false" prices. When they do they encounter quantity constraints, this in turn, as Arrow has noted, stops them acting as perfect competitors. In particular they must form an hypothesis concerning a possibility of affecting their quantity constraints by a change of price. This hypothesis is called a conjecture. A set of prices and quantity signals at which desired trades are achieved and no prices change is advantageous under the conjecture is a conjectural equilibrium. Economies can have conjectural equilibria even when they have a Walrasian one. Naturally one wants a theory of conjecture formation. In what follows it is shown, mainly by examples, that it is not fruitful to look for rational conjectural equilibria (defined in the sequel). One concludes that at best one could hope that agents conjecture Marshallian schedules.
\end{abstract}

\section{Introduction}

In orthodox theory an agent is described by his endowment, tastes and technological production possibilities which are open to him. One does not enquire how these characteristics of the agent came to be what they are, nor, in general, does one allow the characteristics to be affected by the economic environment. The characteristics are arbitrarily given by the history of the economy and of the agent and so, for instance, there are many equilibria depending on the characteristics. In particular if the total endowment of goods is given, any allocation of these between agents (in a pure exchange economy), will be an equilibrium for some tastes and endowment distribution. There does not seem to me anything wrong with the conclusion that equilibria are not history free (they are not independent of initial conditions). Indeed one may adduce rather strong arguments to support the view that history free theorems in the Social Sciences are bogus. To say that the equilibrium set depends on history is not to make equilibrium theory vacuous, the reverse is the case. For one is thereby taking the view that empirical evidence is required to generate interesting propositions. The relevance of these remarks to what follows will be seen below.

* This work was supported by National Science Foundation Grant SOC74-11446 at the Institute for Mathematical Studies in the Social Sciences, Stanford University. 
The orthodox description of the agent is however incomplete on several counts. The most important omission concerns the information available to the agent. For instance if one considers the set of all physical objects in an economy a partition of this set will define the goods which one agent can distinguish. The partition may differ between agents and need not be independent of economic signals. One need only think of second-hand motor cars or different qualities of labour to see that an assumption that all agents have identical fine partitions is not satisfactory. Pari passu the same remarks apply to the partitioning of states of nature; see Radner (1968). A great deal of work has recently been undertaken mainly in the context of very simple models, to study the consequences of enriching the agent's description by endowing him with an information structure and taking account of the possibility that this structure may be only partly a characteristic, i.e. may in part at least depend on economic events (e.g. Rothschild, 1974).

Related to this is the observation that agents have, in general, to deduce their economic environment from the signals which they receive. Thus in orthodox theory the agent does not know the production possibility set of the economy but only relative prices. One of the beautiful aspects of the theory is that this is all he needs to know. Yet even in this simple orthodox world there is a theoretical lacuna: there is no description in terms of the decisions of agents of how prices come to be what they are. It is true that there is a very special account of exchange processes between agents which terminate in allocations which can be supported by competitive prices. But that is hardly satisfactory although even such special constructions may be superior to the auctioneer. For most markets it is simply the case that the description of the agent and of the signals which he receives is not rich enough for a theory of price formation by the agents. I have now come to the central issues to be studied in this paper. Since they are easy to misunderstand I shall discuss them further before introducing technicalities.

The proposal to study an economy which is sufficiently well described to answer the question: "why are the signals received by agents what they are?" is not at all to embark on "dynamics", except in a very weak sense. The weak sense is that an equilibrium must be recognizable as a stationary state of a dynamic system, the finer characteristics of which may be unknown. For instance, in the orthodox tâtonnement matters are normally too complicated to give a precise account of the evolution of prices from a given starting point. But the dynamic equations induce the definition of an equilibrium as stationary points. If the auctioneer is replaced by the agents who change the prices at which they are willing to trade whenever they consider this to be profitable then the stationary point of the dynamic system will have to be a set of signals at which agents do not see profits to be made by changing price. The set of stationary points or equilibria may include those of the tâtonnement but clearly need not coincide with the set of equilibria of the latter. I am 
making the obvious point that the states which we designate as equilibria cannot be independent of the theory of how signals and allocations change. The underlying axiom of the Arrow-Debreu theory is that (at positive prices), prices are stationary iff target excess demand is everywhere zero. If this assumption is changed, and nothing else in the description of the economy is changed, we may expect to find states which previously did not, and now do, qualify as equilibrium states.

At prices which are not in the stationary set of prices for a tâtonnement it is true by definition that not all agents can carry out their intended transactions. One postulates that this gives rise to a further set of signals which tell some of the agents that the transactions which are open to them at these prices are restricted in size. One now requires a theory or rule of the generation of such quantity signals (e.g. a "rationing scheme"), and one requires a theory of the agents' adjustments to these signals. It is in this second stage that one needs the notion of conjectures. If we include in the actions of an agent not only the amounts of each good which he wishes to trade but also the prices which he will announce as those at which he is willing to trade then in the first instance we are looking for a correspondence from the signals received by an agent to the set of actions he conjectures to be available to him. Call it the action correspondence. The equilibrium notion is fairly clear: it is signals received by agents such that the best action for each in the set of possible actions again induces the original signals. A formal definition is found below. Such an equilibrium I want to call a conjectural equilibrium.

I can now return to my opening paragraph. Certainly in the above description of a conjectural equilibrium the designated equilibrium states depend on the conjectures with which we have endowed the agents-e.g. their beliefs of the relation there might be between their ration and their announced price. But the conjectures are unexplained and to that extent conjectural equilibria appear to be arbitrary. As a first reply one is tempted to say that this is no different from the arbitrary tastes of orthodoxy. Certainly this is not entirely unjustified. But there is an objection to this, namely that it may be more convincing to believe that there is no clear inducement to discover which are "correct" tastes. I am not at all sure that this objection has much force. A person brought up on hamburgers may continue with this unpleasing diet even if it is the case that if he tried fish and chips he would discover that he preferred that. Just in the same way a person may continue to find himself unemployed ever so often at a given wage and conjecture wrongly that he can do nothing about it by proposing a lower wage and never undertake the experiment which would reveal this mistake. The belief of the orthodox that given sufficient time men discover their true environment suggests a certain ignorance of both anthropology and history. Children were sacrificed for good harvests for centuries and many people believe that the quantity of money determines the level of money income. Both are wrong conjectures. 
That conjectures may be the outcome of past experience and that they may be "given" for the theorist and discoverable by empirical enquiry is to me acceptable. It is a view which decisively divides both Keynesians and Marxists from orthodoxy. The world is to be explained at least partly by the way agents perceive it and the way in which they perceive it is partly for history, partly for sociology, and partly for psychology. To the orthodox perception does not enter in the story. That is of course why they are so sanguine about the working of the invisible hand.

Nonetheless the orthodox pose an interesting problem when they suggest that the arbitrariness of conjectures be removed by the requirement that they be "correct". As we shall see that requirement is not unambiguously defined and may be impossible to satisfy. In what follows I shall be mainly concerned with that problem.

\section{A Simple Conjectural Economy}

Let there be $H$ households, $F$ firms and $(l+1)$ goods. The generic subscript of an agent is $a$ and $a=h$ refers to a household and $a=i$ to a firm producing good $i$. Each firm produces only one good and each good is produced by a different firm. The subscript $i=0$ refers to leisure. The production sets $Y_{i} \subset R^{l+1}$ of all firms are strictly convex and $y_{i} \in Y_{i}$ is the vector with $y_{i i} \geqslant 0, y_{i 0} \leqslant 0$ and $y_{i j}=0, j \neq i, 0$. Good $i$ is produced by only the input of leisure. All $Y_{i}$ are compact and $y_{i} \in Y_{i}, y_{i i} \neq 0 \rightarrow y_{i 0}<0$. Households have strictly convex closed preferences on $R_{+}^{l+1}$, are endowed with $l_{n 0}>0$ units of leisure (and nothing else) and receive the profit of firms which are distributed among them according to a fixed rule. One writes $x_{h} \in R^{l+1}$ as the demand vector of $h$, $x=\Sigma_{h} x_{h}$. Lastly let $\hat{y} \in X^{l} R^{l+1}$ be the allocation of production among firms.

I shall assume here that households have perfectly competitive conjectures. By this I mean that households choose $x_{h}$ which is best in their preferences from the budget set.

$B_{h}(p, \hat{y})=\left\{x_{h} \mid p x_{h} \leqslant p_{0} l_{h 0}+\Sigma \beta_{h i} p \cdot y_{i}\right\}$

where $0 \leqslant \beta_{h i} \leqslant 1$ each $i$.

By my assumption this gives rise to the demand functions:

$x_{h}=x_{h}(p, \hat{y})$ all $h$.

Let firm $i$ observe the price vector $p \in R_{+}^{l+1}$, the demand $x_{i}$ and a labour ration $L_{i 0}<0$. The latter is a signal that firm $i$ must choose its production at $p$ from $Y_{i} \cap\left\{y_{i} \mid y_{i 0} \geqslant L_{i 0}\right\}$. We stipulate

$\sum_{i} L_{i 0}=\sum_{h}\left(x_{h 0}-l_{h 0}\right)$ 
Now write

$\pi_{i}\left(p, x_{i}, L_{i 0}, y_{i}\right)$

as the conjectured profit function of firm $i$. We postulate the following properties:

$\pi .1 \pi_{i}\left(p, x, L_{i 0}, y_{i}\right)=p \cdot y$ for $y_{i}$ with $y_{i i}=x_{i}, y_{i 0}=L_{، 0}$

$\pi .2 \pi_{i}\left(p, x_{i}, L_{i 0}\right)<p \cdot y_{i}$ for $y_{i i} \geqslant x_{i}, y_{i 0} \leqslant L_{i 0}$ and at least one inequality strict.

$\pi .3 \pi_{i}\left(p, x_{i}, L_{i 0}, y_{i}\right)>p \cdot y_{i}$ for $y_{i i} \leqslant x_{i}, y_{i 0} \geqslant L_{i 0}$ and at least one inequality strict.

$\pi .4$ Given $\left(p, x_{i}, L_{i 0}\right), \pi_{i}$ is concave in $y_{i i}$ and $-y_{i 0}$

Each firm $i$ chooses $y_{i} \in Y_{i}$ given the signal $\left(p, x_{i}, L_{i 0}\right)$ to attain the highest conjectural profit. Notice that $\pi .2$ for instance implies that the firm conjectures that it must sell at a lower price than $p_{i}$ if it wants to produce more than $x_{i}$ and/or buy labour at a higher price than $p_{0}$ if it wants to employ more than $L_{i 0}$. We may write the production choice of firm $i$ as

$y_{i}=y_{i}\left(p, x_{i}, L_{i 0}\right)$

Lastly write $L_{0}=\left\{L_{10} \ldots L_{H 0}\right\}$

D.2.1. We say that $p^{0}, L_{0}^{0},\left(x_{1}^{0} \ldots x_{H}^{0}\right), \hat{y}^{0}$ is a conjectural equilibrium if

(a) $x_{h}^{0} \geqslant_{h} x_{h}$ all $x_{h} \in B_{h}\left(p^{0}, \hat{y}^{0}\right)$ all $h$

(b) $p^{0} y_{i}^{0}=\pi_{i}\left(p^{0}, x_{i}^{0}, L_{i 0}^{0}, y_{i}^{0}\right) \geqq \pi_{i}\left(p^{0}, x_{i}^{0}, L_{i 0}, y_{i}\right) \quad$ all $y_{i} \in Y_{i} \quad$ all $i$

(c) $y_{i i}^{0}=x_{i}^{0} \quad$ all $i=1 \ldots l$

(d) $\sum y_{i 0}^{0}=\sum L_{i 0}^{0} \quad$ when $\quad \sum_{i} L_{i 0}^{0}=\sum_{h}\left(x_{h 0}^{0}-l_{h 0}\right)$

The definition is straightforward. Of course the economy considered is somewhat special in particular in insisting that households are endowed with competitive conjectures. In the sequel I shall follow tradition and not ask that these conjectures of households correspond to what is the case.

Now the profit functions $\pi_{i}(\cdot)$ embody the conjectures of firms and at the moment are arbitrary up to $\pi .1-\pi .4$. In studying the notion of rational conjectures it will be as well not to be too ambitious at the outset. In particular I shall start with considering the possibility of imposing local restrictions on conjectures.

To do this I need to define a conjectural equilibrium relatively to the production of firm $i$. Let $p^{0}, L_{0}^{0},\left(x_{1}^{0} \ldots x_{H}^{0}\right), \hat{y}^{0}$ be a conjectural equilibrium and consider $y_{i} \in N\left(\varepsilon, y_{i}^{0}\right)$ where $N(\cdot)$ is a small, $(\varepsilon)$, neighbourhood of $y_{i}^{0}$ in $R^{l+1}$. Then 
D.2.2. Let $p\left(y_{i}\right), L_{0}\left(y_{i}\right),\left(x_{1}\left(y_{i}\right) \ldots x_{H}\left(y_{i}\right)\right), \hat{y}\left(y_{i}\right)$ be called $^{\mathbf{1}}$ a conjectural equilibrium relatively to $y_{i} \in N\left(\varepsilon, y_{i}^{0}\right)$ if

a) $x_{h}\left(y_{i}\right) \geqslant_{h} x_{h}$ all $\left.x_{h} \in B_{h}\left(p\left(y_{i}\right), \hat{y}\left(y_{i}\right)\right)\right\}$ all $h$

b) $p\left(y_{i}\right) y_{k}\left(y_{i}\right)=\pi_{k}\left(p\left(y_{i}\right), x_{k}\left(y_{i}\right) !^{!} L_{k 0}\left(y_{i}\right), y_{k}\left(y_{i}\right)\right) \geqslant \pi_{k}\left(p\left(y_{i}\right), x_{k}\left(y_{i}\right), \quad L_{k 0}\left(y_{i}\right), y_{k}\right)$ all $y_{k} \in Y_{k}$ and all $k \neq i$.

c) $y_{k k}\left(y_{i}\right)=x_{k}\left(y_{i}\right)$ all $k=1 \ldots l$

(d) $\sum_{k} y_{k 0}\left(y_{i}\right)=\sum_{k} L_{k 0}\left(y_{i}\right)$ and $\sum_{k} L_{k 0}\left(y_{i}\right)=\sum_{h}\left(x_{h 0}\left(y_{i}\right)-l_{k 0}\right)$

It will be seen that the difference between a conjectural equilibrium and a conjectural equilibrium relatively to $y_{i}$ is that in the former we do, and in the latter we do not, demand that firm $i$ should have maximum profits under its conjectures. The reason for this construction will become clear almost at one.

Let $E_{i}\left(y_{i}, y_{i}^{0}, \varepsilon\right) \subset R^{l+1} \times R^{H} \times R_{+}^{H(l+1)} \times R^{(F \cdot 1)(l+1)}$ be the set of conjectural equilibria relative to $y_{i}$ when $y_{i} \in N\left(y_{i}^{0}, \varepsilon\right)$. An element of $E_{i}(\cdot)$ is $\left(p\left(y_{i}\right), L\left(y_{i}\right)\right.$, $\left(x_{1}\left(y_{1}\right) \ldots x_{H}\left(y_{i}\right), \hat{y}\left(y_{i}\right)\right)$. I write $E_{i p}(\cdot)$ as the projection of $E_{i}(\cdot)$ onto the price space.

D.2.3. I call $\left(p^{0}, x_{1}^{0} \ldots x_{H}^{0}, L_{0}^{0}, y^{0}\right)$ an $\varepsilon$-reasonable conjectural equilibrium if for all $i=1 \ldots e$ and given $\varepsilon$

either a) $p_{i} y_{i} \leqslant \pi_{i}\left(p^{0}, x_{i}^{0} L_{i 0}^{0}, y_{i}^{0}\right), p_{i} \in E_{i p}\left(y_{i}, y_{i}^{0}, \varepsilon\right), y_{i} \in Y_{i} \cap N\left(g_{i}^{0}, \varepsilon\right)$

or b) $E_{i}\left(y_{i}, y_{i}^{0}, \varepsilon\right)$ is empty.

Let me explain the idea of D.2.3. One considers a given conjectural equilibrium and asks what would happen to the equilibrium profits of a firm $i$ if it deviated slightly in its production plan from what, under its conjectures is an optimum plan. If such a slight deviation is inconsistent, given the conjectures of all other firms with an equilibrium (D.2.3.b), we argue that it is reasonable for firm $i$ not to undertake that deviation. If it is consistent with such an equilibrium but profits are no higher for firm $i$ than they were without that deviation (D.2.3.a) we also argue that the firm $i$ is reasonable in not making the deviation. In this of course D.2.3.b is the least attractive. But unless one is willing to open the Pandora's box of dynamics there is not much alternative.

I use the terminology 'reasonable' to distinguish the case where a firm may be right in its belief that it cannot locally improve profits for the 'wrong' reason from that where the firm is right in that belief for the 'right' reason (which I shall call 'rational'). Thus a firm may wrongly predict the relevant elements of $E_{i}(\cdot)$ and yet be correct in its conclusion that it cannot improve itself by small changes in production. A more stringent requirement is that in a conjectural equilibrium each firm $i$ should correctly predict the element of $E_{i}(\cdot)$. Thus

1 The $i$ th vector of $\hat{y}\left(y_{i}\right)$ is $y_{i}$. 
D.2.4. I call $\left(p^{0}, x_{1}^{0} \ldots x_{H}^{0}, L^{0}, \hat{y}^{0}\right)$ an $\varepsilon$-rational conjectural equilibrium if

a) If is an $\varepsilon$-reasonable equilibrium

and b) For all $i$ and $y_{i} \in Y_{i} \cap N\left(\varepsilon, y_{i}^{0}\right)$

$$
p_{i} y_{i}=\pi_{i}\left(p, x_{i}, L_{i 0}, y\right) \quad \text { where }\left(p, x_{1}, \ldots, x_{H}, L_{0}, \hat{y}\right) \in E_{i}\left(y_{i}, y_{i}^{0}\right) \text {. }
$$

Thus in an $\varepsilon$-rational conjectural equilibrium not only is there no other conjectural equilibrium consistent with $y_{i} \in N\left(y_{i}^{0}, \varepsilon\right)$ in which $i$ 's profits are higher than they are in the given equilibrium, but the profits attained in any conjectural equilibrium relatively to $y_{i}$ are those which $i$ 's conjectures predict.

Both of the definitions of $\varepsilon$-reasonable and $\varepsilon$-rational conjectural equilibrium are in the general equilibrium spirit and it may be thought that they are too demanding even before one has considered the problem of their existence. So let me consider one last alternative formulation which is more in the spirit of Nash equilibria.

Let $\hat{y}_{N}^{0}\left(y_{i}\right)$ be the vector $\hat{y}^{0}$ with $y_{i}$ replacing $y_{i}^{0}$. (The subscript $N$ reminds us of the Nash feature that the production vectors of firms other than $i$ are fixed.) We require the notion of an $\varepsilon$-Nash deviation from a conjectural equilibrium relatively to $y_{2}$

D.2.5. Let $\left(p^{0}, x_{1}^{0}, \ldots, x_{H}^{0}, L_{0}^{0}, \hat{y}^{0}\right)$ be a conjectural equilibrium. Then $p^{N}\left(y_{t}\right) \epsilon$ $R_{+}^{l+1}, x_{h}^{N}\left(y_{i}\right) \in R_{+}^{l+1},(h=1, \ldots, H), L_{0}^{N}\left(y_{i}\right) \in R^{H}$ is called an $\varepsilon-N a s h$ deviation from the given conjectural equilibrium relatively $y_{i}$ if when $y_{i} \in Y_{i} N\left(\varepsilon, y_{i}^{0}\right)$ :

(a) $x_{h}^{N}\left(y_{i}\right) \geqslant_{h} x_{h} \quad$ all $x_{h} \in B_{h}\left(p^{N}\left(y_{i}\right), \hat{y}_{N}^{0}\left(y_{i}\right)\right)$ all $h$

(b) $\sum_{h} x_{h k}^{N}\left(y_{i}\right)=y_{k k}^{0} \quad$ all $k \neq i$

$$
\begin{aligned}
& \sum_{h} x_{h i}^{N}\left(y_{i}\right)=y_{i i} \\
& \sum_{i}\left(x_{h 0}^{N}\left(y_{i}\right)-l_{h 0}\right)=\sum L_{0 k}^{N}\left(y_{i}\right)=\sum_{k \neq i} y_{k 0}^{0}+y_{i 0}
\end{aligned}
$$

Thus in an $\varepsilon$-Nash deviation we calculate the equilibrium of the economy on the supposition that all firms other than $i$ keep their productions as it is in conjectural equilibrium and therefore ignore the fact that this constancy of production may not be profit maximising for these firms under their conjectures. One may say that this is an interesting concept either because no firm $i$ could calculate anything more elaborate or, more speculatively that for a small enough firm $i$ at $\varepsilon$, the $\varepsilon$-Nash deviation is a good approximation to an $\varepsilon$-conjectural equilibrium relatively to $y_{i}$. One now has

D.2.6. The conjectural equilibrium $\left(p^{0}, x_{1}, \ldots, x_{H}^{0} L_{0}^{0}, \hat{y}^{0}\right)$ is $\varepsilon$-Nash rational if for all $i$ and $y_{i} \in Y_{i} \cap N\left(\varepsilon, y_{i}\right)$ there exists an $\varepsilon$-Nash deviation relatively to $i$ such that

$p^{N}\left(y_{i}\right) y_{i}=\pi_{i}\left(p^{N}\left(y_{i}\right), x_{i}^{N}\left(y_{i}\right), L_{0 i}^{N}\left(y_{i}\right), y_{i}\right)$ 
That is, in an $\varepsilon$-Nash rational conjectural equilibrium the conjectural profit functions of firms correctly predict the profits to be made from an $\varepsilon$-Nash deviation.

We now have a good many candidates for tying down conjectures. One wants to ask two questions: (i) are there good economic grounds for supposing conjectural equilibria to have one or more of the characteristics captured by $\varepsilon$-reasonable, $\varepsilon$-rational and $\varepsilon$-Nash rational? and (ii) are all of these equilibria non-vacuous - that is, could they exist? Until we have explored (ii) it is not worthwhile arguing about (i).

\section{Existence Problems}

Given the assumptions in Section II one can show that a conjectural equilibrium exists ${ }^{1}$ and I shall here take this for granted. To proceed to the next task it will be convenient to simplify the model in the non-essential way of letting firms have competitive conjectures in the labour market. That is we now remove the quantity signals $L_{i 0}$ from the profit functions. I shall also suppose that a conjectural equilibrium with $p_{0}>0$ exists and henceforth, without change of notation, take $p_{0}=1$ in $p$. Lastly I postulate that all functions that interest me are of class $C^{2}$.

Let $\hat{\pi}=\left(\pi_{1}, \ldots, \pi_{l}\right)$ be the vector of profits $p \cdot y_{i}$. Let us also now write

$y_{i 0}=f_{i}\left(y_{i i}\right)$

for the input of labour required to produce $y_{i i}$. One takes $f_{i}(\cdot)$ as convex. From what has already been said one may write

$x_{i}=x_{i}(p, \hat{y})$

and so

$x_{i}-y_{i i} \equiv g_{i}(p, \hat{y})$

where without change of notation I now take $\hat{y} \in R_{+}^{l}$ to be the vector of outputs. Also if $\pi_{i}\left(p_{i}, x_{i}, y_{i i}\right)$ is the conjectural profit function, let

$h_{i}\left(p_{i}, x_{i}, y_{i i}\right) \equiv \bar{h}_{i}(p, \hat{y}) \equiv \frac{\partial \pi_{i}}{\partial y_{i i}}$

Then $\left(p^{0}, \hat{y}^{0}\right)$ is a conjectural equilibrium if

a) $g\left(p^{0}, y^{0}\right)=0$

b) $\left.\bar{h}\left(p^{0}, y^{0}\right)=0\right\}$

1 A proof for closely related models will be found in Hahn (forthcoming) and Negishi (1968). 
Suppose that $\left(\mathrm{p}^{0}, \hat{y}^{0}\right)>0$ and let the superscript 0 to a function denote that it is evaluated at $\left(p^{0}, \hat{y}^{0}\right)$. Then the conjectural equilibrium is called regular if

$$
M\left(p^{0}, \hat{y}^{0}\right)=\left[\begin{array}{ll}
g_{p}^{0} & g_{y}^{0} \\
\tilde{h}_{p}^{0} & \hbar_{y}^{0}
\end{array}\right]
$$

is of full rank (i.e. of rank $2 l$ ).

It will be convenient to state the regularity condition in a different form. Let $H$ always denote an $l \times l$ diagonal matrix where

$\left.H_{p}^{0}=\left[h_{i p_{i}}^{0}\right], H_{x}^{0}=\left[h_{i x_{i}}^{0}\right], H_{y}^{0}=\left[h_{i y_{i i}}^{0}\right] . H_{z}^{0}=h_{i x_{i}}^{0}+h_{i y_{i i}}^{0}\right]$

Then

$\hbar_{p}^{0}=H_{p}^{0}+H_{x}^{0} x_{p}=H_{p}^{0}+H_{x}^{0} g_{p}^{0}$

$\hbar_{y}^{0}=H_{y}^{0}+H_{x}^{0} x_{y}=H_{y}^{0}+H_{x}^{0}+H_{x}^{0}\left[x_{y}-I\right]=H_{y}^{0}+H_{x}^{0}+H_{x}^{0} g_{y}^{0}$

Hence if $M\left(p^{0}, \hat{y}^{0}\right)$ is non-singular so is $M^{*}\left(p^{0}, \hat{y}^{0}\right)$ where

$M^{*}\left(p^{0}, \hat{y}^{0}\right)=\left[\begin{array}{ll}g_{p}^{0} & g_{y}^{0} \\ H_{p}^{0} & H_{z}^{0}\end{array}\right]$

D.3.1. A conjectural equilibrium $\left(p^{0}, \hat{y}^{0}\right)$ will be called regular if $\left(p^{0}, \hat{y}^{0}\right)>0$ and $M^{*}\left(p^{0}, \hat{y}^{0}\right)$ is of full rank.

Now consider a regular conjectural equilibrium and take a small variation in the production of firm $k$. We want to find the conjectural equilibrium (if it exists), relatively to $\left(y_{k k}^{0} \pm \varepsilon\right)$ where $\varepsilon$ is very small. Suppose this conjectural equilibrium is $(p, \hat{y})$. Then it must satisfy:

$\left.\begin{array}{c}g_{p}^{0}\left(p-p^{0}\right)+g_{y}^{0}\left(\hat{y}-\hat{y}^{0}\right)=0 \\ \text { and } \bar{h}_{i p}^{0}\left(p-p^{0}\right)+\bar{h}_{i y}^{0}\left(\hat{y}-\hat{y}^{0}\right)=0 \quad \text { all } i=k\end{array}\right\}$

Also one has $y_{k k}^{0}\left(p_{k}-p_{k}^{0}\right)+\left(p_{k}^{0}+f_{k}^{\prime}\left(y_{k k}^{0}\right)\right)\left(y_{k k}-y_{k k}^{0}\right)=\pi_{k}-\pi_{k}^{0}$

So if $H_{p}^{0}(k)$ is the matrix $H_{p}^{0}$ with its $k$ th diagonal element replaced by $y_{k k}^{0}$ and $H_{z}^{0}(k)$ is the matrix $H_{z}^{0}$ with its $k$ th diagonal element replaced by $\left(p_{k}^{0}+f_{k}^{\prime}\left(y_{k}^{0} k\right)\right)$ one is interested in the equations

$M_{k}^{*}\left(p^{0}, y^{0}\right)\left(p-p^{0}, y-y^{0}\right)=\left\{\delta_{i k+l}\left(\pi_{k}-\pi_{k}^{0}\right)\right\}$

where

$M_{k}^{*}\left(p^{9}, \hat{y}^{0}\right)=\left[\begin{array}{ll}g_{p}^{0} & g_{y}^{0} \\ H_{p}^{0}(k) & H_{z}^{0}(k)\end{array}\right]$

Proposition 3.1. A necessary and sufficient condition for a regular conjectural equilibrium to be $\varepsilon$-rational for $\varepsilon$ arbitrarily small is that either the matrix 
$M_{K}^{*}\left(p^{0}, \hat{y}^{0}\right)$ be singular for all $k=1, \ldots, l$ or that no equilibria relatively to $\left(y_{k k}^{0} \pm \varepsilon\right)$ exist for any ${ }^{1} k$.

Proof. (a) Necessity. If $\left(p^{0}, \hat{y}^{0}\right)$ is a conjectural equilibrium and $M_{k}^{*}\left(p^{0}, \hat{y}^{0}\right)$ is of full rank for some $k$ then 3.4 has a solution with $\pi_{k}>\pi_{k}^{0}$ so that the equilibrium would not be $\varepsilon$-reasonable and so not $\varepsilon$-rational.

(b) Sufficiency. If at $\left(p^{0}, \hat{y}^{0}\right)$ the condition of the proposition is satisfied then one can solve

$M_{k}^{*}\left(p^{0}, \hat{y}^{0}\right)\left(p-p^{0}, \hat{y}-\hat{y}^{0}\right)=\{0\}$

for all the conjectural equilibria $(p, \hat{y})$ relatively to $\left(y_{k k}^{b} \pm \varepsilon\right)$. In all such equilibria $\pi_{k}=\pi_{k}^{0}$ and there exists no such equilibrium with $\pi_{k}>\pi_{k}^{0}$.

The proposition is of course trivial-indeed it is almost a definition of an $\varepsilon$-rational conjectural equilibrium. Nonetheless it is of some help with the rather intractable problem of whether $\varepsilon$-rational equilibria exist. To see this $I$ consider an example.

Suppose that the conjectural profit function of each firm is derived from the conjectural inverse demand function:

$p_{i}+\beta_{i}\left(y_{i i}-x_{i}\right) . \quad \beta_{i}<0$ all $i$

Also assume that

$f_{i}\left(y_{i i}\right)=-c_{i} y_{i i} \quad c_{i}>0$ all $i$

Then one verifies that $H_{p}^{0}=I, H_{z}^{0}=\left\{\beta_{i}\right\}$ while $H_{p}^{0}(k)$ has $y_{k k}^{0}$ in the $k$ th diagonal place and $H_{z}^{0}(k)$ has $-\beta_{k} y_{k k}^{0}$ as the $k$ th diagonal element.

Let us consider only regular conjectural equilibria for this economy so that writing small letters for the determinant of the matrix, $m^{*}(p, \hat{y}) \neq 0$ at any conjectural equilibrium $(p, \hat{y})$. In the present economy one has

$m_{l+k, k}^{*}+\beta_{k} m_{l+k, l+k}^{*}=m^{*}$

where I now omit the arguments $(p, \hat{y})$ and where $m_{i j}^{*}$ are co-factors in the usual notation. If the conjectural equilibrium is $\varepsilon$-rational and we use Proposition 3.1 in the present case one must have

$m_{l+k, k}^{*}-\beta_{k} m_{l+k, l+k}^{*}=0$

and since this must hold for all $k$ one now has

$m_{l+k, k}^{*}=\frac{1}{2} m^{*} \quad$ all $k$

Now let us specialise somewhat further by assuming (i) $l=2$. (ii) all households have parallel linear Engel curves and (iii) labour is supplied inelastically and leisure does not enter the utility function.

1 I shall henceforth in this section ignore the second contingency. Throughout I am strictly concerned with "infinitesimal rationality", i.e. with $\varepsilon \rightarrow 0$. But the exposition will serve if sufficient regularity is granted. 
It is a consequence of (ii) that $g_{p}$ becomes a matrix of substitution terms which in view of (iii) is singular. Also a typical element of $g_{y}$ is $x_{i y_{i}}-\delta_{i j}$. If $\mu$ is the sum of profits and wages then

$x_{i y_{i}}=x_{i \mu}\left(p_{j}-c_{j}\right)=-\beta_{j} x_{i \mu} y_{j j}$

by (iii) where $x_{i \mu}=\partial x_{i} / \partial \mu$. Also by (iii) $\Sigma p_{i} x_{i \mu}=1$. Using all of this and 3.7 one finds that we require of a regular conjectural equilibrium that ${ }^{1}$

(A) $c_{1} \beta_{2} S_{12}+c_{2} \beta_{1} s_{1}=0$

where $S_{i j}$ is a substitution term. From this and (iii) one has

$\frac{c_{1} \beta_{2}}{p_{2}}=\frac{c_{2} \beta_{1}}{p_{1}^{0}}=k$

We also require

(B) $x_{1 \mu}\left[\beta_{1} c_{2} x_{1}-\beta_{2} c_{1} x_{2}\right]+c_{2}=0$

or using 3.8

$k x_{1} \mu\left[p_{1}^{0} x_{1}-p_{2}^{0} x_{2}\right]+c_{2}=0$

These two conditions must be satisfied if the conjectural equilibrium is to be $\varepsilon$-rational. But now suppose the common utility function to be CobbDouglas with exponents $\alpha_{i}(i=1,2)$. Then 3.9 becomes

$k \mu x_{i \mu}\left[\alpha_{1}-\alpha_{2}\right]+c_{2}=0$

Since one wants $k<0$ it now follows that should $\alpha_{2}>\alpha_{1}$ no $\varepsilon$-rational regular conjectural equilibrium exists.

As a second example consider profit conjectures based on the following conjectural inverse demand functions:

$p_{i}\left(y_{i i} / x_{i}\right)^{\beta_{i}}$ where $-1<\beta_{i}<0$

Then one verifies that $H_{p}^{0}=\left\{1+\beta_{i}\right\}, H_{z}^{0}=\{0\}, H_{p}^{0}(k)$ has $y_{k k}^{0}$ in the $k$ th diagonal place and $H_{z}^{0}$ has $-\beta_{k} p_{k}^{0}$. We now assume that $c_{1}=c_{2}=c$ and continue the assumptions (i) to (iii) of the previous example.

The rather tedious manipulations will be found in the appendix. Using Proposition 3.1 one shows that if a given conjectural equilibrium is to be $\varepsilon$-rational one requires

$-\frac{\sigma_{21}}{\sigma_{11}}=\frac{\beta_{1}\left(1+\beta_{2}\right)}{\beta_{2}\left(1+\beta_{1}\right)}$

1 See Appendix for manipulations.

Scand. J. of Economics 1977 
and also that

$-\frac{\sigma_{21}}{\sigma_{11}}>1$

where $\sigma_{i j}$ is the compensated elasticity of demand for good $i$ for a change in the price of good $j$. From elementary theory then 3.12 can also be written as $\frac{x_{1}^{0}}{x_{2}^{0}}>1$

From 3.11 also $\left|\beta_{1}\right|>\left|\beta_{2}\right|$ and so since $p_{i}^{0}\left(1+\beta_{i}\right)=c i=1,2$ one has $p_{1}^{0}>p_{2}^{0}$

But now it is easy to find a hypothesis which makes the fulfilment of these conditions impossible. For instance let the common strictly quasiconcave function be homothetic with the indifference curves having a unitary slope where they cross the $45^{\circ}$ line. Then 3.13 and 3.14 together are not possible.

If one has enough patience one can construct other 'well behaved' economies which have no regular $\varepsilon$-rational conjectural equilibrium. Thus one can relatively easily find examples with three goods where the requirements of Proposition 3.1 conflict with the concavity of the conjectured profit function.

The examples suggest that there will be considerable difficulty in describing an economy with simple enough conjectures which also possesses an $\varepsilon$-rational equilibrium. My present view is that the conditions will turn out to be sufficiently restrictive to make the result uninteresting. But there is another lesson which seems important.

In the examples I gave conjectures a particular form. Now if one takes $\varepsilon$ small enough what one is roughly concerned with is the existence of an equilibrium where the conjectured slope of the inverse demand curve at the equilibrium output accurately predicts the price in an equilibrium relatively to a very small deviation in a producer's output. But as we have seen this will depend not only on the conjectured slopes of other producers but also on their rates of change (the terms $h_{p}, h_{y}$ ). In the examples, in trying to discover whether conjectured slopes can be 'tied down' by asking that they be 'correct' in a proper sense we made these higher order terms arbitrary. So even had our conclusion been positive we should have shown that there exists an equilibrium in which conjectured slopes are 'correct' only at the cost of arbitrarily imposing a form on the conjectured demand function. Indeed I think that under fairly general assumptions one may be able to show that there always exists a conjectural equilibrium with "correct" slopes provided one can arbitrarily specify the form of the conjecture. But this means that we do not escape the arbitrariness of conjectures.

Evidently there are quite hard technical problems and they require further 
investigation. But $I$ think there is enough evidence in this section to warrant the preliminary conclusion that $\varepsilon$-rationality is not a hopeful way of avoiding the arbitrariness-i.e. the exogenous, nature of conjectures.

\section{Nash $\varepsilon$-Rationality}

I shall be brief in this section for the matter requires more investigation than I can give it here. Indeed I shall only consider an example of $l=\mathbf{2}$.

Suppose that $\left(p^{0}, \hat{y}^{0}\right)$ is a conjectural equilibrium and consider a small $\varepsilon$ deviation from $y_{11}^{0}$ by producer one. We are in the first instance interested in the equilibria (if they exist), relatively to that deviation on the supposition that the output of producer two stays at $y_{22}^{0}$. Notice that such equilibria will not in general be conjectural equilibria since producer two will not be maximising relatively to his conjectures. Now $p$ will be an equilibrium relatively to $\left(y_{11}^{0} \pm \varepsilon\right)$ when $\varepsilon$ is small enough if it satisfies

$g_{p}^{0}\left(p-p^{0}\right)=-g_{y_{1}}^{0}\left(y_{11}-y_{11}^{0}\right)$

where $g_{p}^{0}$ has the usual meaning and $g_{y_{1}}^{0} \in R^{2}$ is the vector $\left(x_{1 y_{1}}-1, x_{2 y_{1}}\right)$.

This equation can be solved, when $\left(y_{11}-y_{11}^{0}\right) \neq 0$ iff $g_{p}^{0}$ is not singular. But in one of our examples where households supply labour inelastically and where they ave parallel linear Engel curves, $g_{p}^{0}$ is a singular matrix of substitution terms. Let us call an economy where households satisfy these assumptions Hicksian (cf. Arrow \& Hahn, 1971). Then

Proposition 4.1. Any conjectural equilibrium of a Hicksian economy where labour is supplied inelastically is $\varepsilon$-Nash rational for $\varepsilon$ small enough.

This result is a direct consequence of our general decision to call conjectures rational if deviations in the actions of one agent are not compatible with equilibrium (D.2.3.b). Of course, this is open to argument. Yet it is not easy to see what alternative route should be followed in this case. One could, for instance, relax the purely Walrasian equilibrium notion (and consider equilibria with rationing) or one could try to model the 'true' dynamics of the economy which would give an answer to the agent's question: what will be the case if $\mathrm{I}$ deviate slightly from my present actions? But 'not only is this procedure technically and conceptually hard; it is one which makes it even more impossible to suppose that agents can carry out correct calculations.

So let me now suppose that $g_{p}^{0}$ is not singular which $I$ achieve in the Hicksian economy by dropping the assumption that labour is inelastically supplied (i.e. that it does not enter the utility function). Let

$N_{1}^{0}=\left[\begin{array}{cc}g_{p}^{0} & g_{y_{1}}^{0} \\ y_{11}^{0} & p_{1}^{0}-c_{1}\end{array}\right]$ 
Then if the conjectural equilibrium is $\varepsilon$-Nash rational one wants $N_{1}^{0}$ to be singular. The argument here is exactly as in the previous section the only difference being that $y_{22}=y_{22}^{0}$ by construction.

To see the difficulties one may now encounter let the common utility function be Cobb-Douglas with exponents $\alpha_{i}(i=0 \ldots 2)$ where the subscript 0 refers to leisure. Suppose further that conjectures have the form of 3.9, and choose units so that $c_{i}=1, i=1,2$.

Then the condition that $N_{1}^{0}$ be singular reduces in this special case to

$\alpha_{0}+\alpha_{1} \alpha_{2}-\left(1-\alpha_{2}\right) \alpha_{1}=\frac{1+\beta_{1}}{\beta_{1}} \frac{\left(\alpha_{2}-1\right)}{\alpha_{1}}$

For $\pi .1-\pi .4$ to hold one wants $\beta,<0$ and hence

$\frac{\alpha_{0}}{1-2 \alpha_{2}}>\alpha_{0}$

If 4.1 is violated then no $\varepsilon$-Nash rational conjectural equilibrium is possible. For with $\beta_{1}>0$ no profit maximising choice of the firm exists while for $\beta_{1}<0$, $\mathrm{N}_{1}^{0}$ cannot vanish.

Once again it is rather doubtful that one can find sufficiently general conditions which would ensure the existence of an $\varepsilon$-Nash rational conjectural equilibrium. But this is a matter for further study.

\section{Some Conclusions}

To a practical economist it will be no surprise that the notions of rationality in conjectures explored here are very unpromising. Indeed he would argue that the questions are incorrectly formulated. For either the typical firm is 'small' and hence one should ignore the general equilibrium repercussions of its own actions or it is significant in which case the proper approach is either game theoretic or a rule of thumb.

There evidently is some force in this objection. On the other hand unless a firm is so small (strictly of measure zero), as to make a perfectly competitive conjecture $\varepsilon$-rational in my sense, it will make mistakes when it acts on a conjectured demand curve derived from partial equilibrium hypotheses. By this I mean that it correctly calculates the slope of the demand curve on the hypothesis that all other prices and outputs other than its own are fixed. The question of course is whether these mistakes are small enough to be 'sensibly' ignored.

With sufficient assumptions (which certainly must exclude the CobbDouglas utility function) one can almost certainly establish the existence of a "Marshallian $\varepsilon$-conjectural equilibrium". That would be an equilibrium in which producers have chosen optimally relatively to observed demand and 
price and have correctly calculated the slope of their demand curve at this point on the assumption that all other prices and outputs remain constant. There will be some technical difficulties since one will not be sure without special hypotheses that conjectural profit functions are concave everywhere. But there certainly is a class of utility for which this will be true. Such equilibria will not be $\varepsilon$-reasonable or $\varepsilon$-rational. Hence in an actual experiment a firm may discover that it is mistaken. Depending on cross-elasticities these mistakes may be 'large' for a 'small' firm.

All of this requires further study and the present paper is no more than an introduction to some of the questions which arise. My present view is that if it will prove possible to make conjectures less arbitrary it will have to be done in a Marshallian way. This is not a conclusion congenial to a general equilibrium man.

\section{Appendix}

Linear Conjectures

$m^{*}=\left|\begin{array}{llll}S_{11} & S_{12} & x_{1 \mu}\left(p_{1}-c_{1}\right)-1 & x_{1 \mu}\left(p_{2}-c_{2}\right) \\ S_{21} & S_{22} & x_{2 \mu}\left(p_{1}-c_{1}\right) & x_{2 \mu}\left(p_{2}-c_{2}\right)-1 \\ 1 & 0 & \beta_{1} & 0 \\ 0 & 1 & 0 & \beta_{2}\end{array}\right|$

Adding $p_{1}$ times first row to $p_{2}$ times second row gives

$m^{*}=\left|\begin{array}{llll}S_{11} & S_{12} & x_{1 \mu}\left(p_{1}-c_{1}\right)-1 & x_{1 \mu}\left(p_{2}-c_{2}\right) \\ 0 & 0 & -c_{1} & -c_{2} \\ 1 & 0 & \beta_{1} & 0 \\ 0 & 1 & 0 & \beta_{a}\end{array}\right|$

Let $\Delta$ be the top right-hand $2 \times 2$ determinant. Then

$m^{*}=S_{11} \beta_{1} c_{2}-S_{12} \beta_{2} c_{1}+\Delta$

$m_{31}^{*}=-S_{12} c_{1} \beta_{2}+\Delta$

$m_{41}^{*}=S_{11} c_{2} \beta_{1}+\Delta$

From 3.7: $m_{31}^{*}=m_{41}^{*}$ gives

$S_{11} c_{1} \beta_{1}+S_{12} c_{1} \beta_{2}=0$

which is $A$ of the text. For 3.7 also $2 m_{31}^{*}=m^{*}$ or

$S_{11} \beta_{1} c_{2}+S_{12} \beta_{2} c_{1}-\Delta=0$

Scand. J. of Economics 1977 
and so

$\Delta=c_{2}-c_{2} x_{1 \mu}\left(p_{1}-c_{1}\right)+c_{1} x_{1 \mu}\left(p_{2}-c_{2}\right)=c_{2}+\left[c_{2} \beta_{1} x_{1}-c_{1} \beta_{2} x_{2}\right]=0$

which is $B$ of the text.

\section{Constant Elasticity Conjectures}

We have

$M^{*}=\left[\begin{array}{ll}g_{p}^{0} & g_{y}^{0} \\ \left\{1+\beta_{i}\right\} & 0\end{array}\right]$

whence

$m^{*}=\prod^{i}\left(1+\beta_{i}\right)\left|g_{y}^{0}\right|$

Proceeding as in the previous example and using $p_{k}^{0}-c=-\beta_{k} p_{k}^{0}$ one finds

$\left|g_{y}^{0}\right|=x_{1 \mu} \beta_{1} c p_{1}^{0}-\beta_{2} c p_{2}^{0}+c$

Also

$\left[\begin{array}{llll}g_{p_{1}}^{0} & g_{p_{2}}^{0} & g_{y_{1}}^{0} & g_{y_{2}}^{0} \\ y_{11}^{0} & 0 & -\alpha_{1} p_{1}^{0} & 0 \\ 0 & \left(1+\beta_{2}\right) & 0 & 0\end{array}\right]$

and similarly for $M_{2}^{*}$. One finds

$m_{1}^{*}=\left(1+\beta_{2}\right)\left[x_{1}^{0}\left|g_{y}^{0}\right|-\beta_{1} p_{1}^{0} m_{33}^{*}\right]$

$m_{2}^{*}=\left(1+\beta_{1}\right)\left[x_{2}^{0}\left|g_{y}^{0}\right|-\beta_{2} p_{2}^{0} m_{44}^{*}\right]$

Calculating further yields

$m_{33}^{*}=-S_{11}\left(1+\beta_{2}\right) c, \quad m_{44}^{*}=S_{12}\left(1+\beta_{1}\right) c$

By Proposition 3.1 one wants $m_{1}^{*}=m_{2}^{*}=0$ if the equilibrium is to be $\varepsilon$-rational. So

$\beta_{1} p_{1}^{0} m_{33}^{*} / x_{1}^{0}=\beta_{2} p_{2}^{0} m_{44}^{*} / x_{2}^{0}$

or by (A.2)

$-\beta_{1}\left(1+\beta_{2}\right) c \sigma_{11}=\beta_{2}\left(1+\beta_{1}\right) c \sigma_{12}$

which then yields 3.11 of the text.

Next since $m_{33}^{*}>0, m_{44}^{*}>0, \beta_{i}>0$ one must have

$\left|g_{y}^{0}\right|<0$. 
So from (A.1)

$\frac{\beta_{1} p_{1}^{0}}{\beta_{2} p_{2}^{0}}=\frac{\beta_{1}\left(1+\beta_{2}\right)}{\beta_{2}\left(1+\beta_{1}\right)}>1$

This confirms 3.12 of the text.

\section{References}

Arrow, K. J. \& Hahn, F. H.: General competitive analysis. Holden Day, 1971.

Hahn, F. H.: On non-Walrasian equilibria. Review of Economic Studies (forthcoming).

Negishi, Y.: Monopolistic competition and general equilibrium. Review of Economic Studies, 1968.
Radner, R.: Competitive equilibrium under uncertainty. Econometrica, 1968.

Rothschild, M.: A two-armed-bandit theory of market pricing. Journal of Economic Theory, 1974. 